\title{
MS-Rescue: A Computational Pipeline to Increase the Quality and Yield of Immunopeptidomics Experiments
}

Andreatta, Massimo; Nicastri, Annalisa; Peng, Xu; Hancock, Gemma; Dorrell, Lucy; Ternette, Nicola; Nielsen, Morten

\section{Published in:}

Proteomics

Link to article, DOI:

10.1002/pmic.201800357

Publication date:

2019

Document Version

Early version, also known as pre-print

Link back to DTU Orbit

Citation (APA):

Andreatta, M., Nicastri, A., Peng, X., Hancock, G., Dorrell, L., Ternette, N., \& Nielsen, M. (2019). MS-Rescue: A Computational Pipeline to Increase the Quality and Yield of Immunopeptidomics Experiments. Proteomics, 19(4), [1800357]. https://doi.org/10.1002/pmic.201800357

\section{General rights}

Copyright and moral rights for the publications made accessible in the public portal are retained by the authors and/or other copyright owners and it is a condition of accessing publications that users recognise and abide by the legal requirements associated with these rights.

- Users may download and print one copy of any publication from the public portal for the purpose of private study or research.

- You may not further distribute the material or use it for any profit-making activity or commercial gain

- You may freely distribute the URL identifying the publication in the public portal 


\title{
MS-rescue: A Computational Pipeline to Increase the Quality and Yield of Immunopeptidomics Experiments
}

\author{
Massimo Andreatta ${ }^{1}$, Annalisa Nicastri ${ }^{2}, \mathrm{Xu} \mathrm{Peng}^{2}$, Gemma Hancock $^{2}$, Lucy Dorrell ${ }^{2,3}$, \\ Nicola Ternette ${ }^{4}$, Morten Nielsen ${ }^{1,5}$ \\ ${ }^{1}$ Instituto de Investigaciones Biotecnológicas, Universidad Nacional de San Martín, San Martín, \\ Argentina \\ ${ }^{2}$ Nuffield Department of Medicine, University of Oxford, United Kingdom \\ ${ }^{3}$ Oxford NIHR Biomedical Research Centre, United Kingdom \\ ${ }^{4}$ The Jenner Institute, University of Oxford, United Kingdom \\ ${ }^{5}$ Department of Bio and Health Informatics, Technical University of Denmark, Lyngby, Denmark
}

\section{Corresponding author:}

Morten Nielsen

Phone: +45 45252425

Fax: +4545931585

Email: mniel@bioinformatics.dtu.dk

Keywords: peptidome, mass spectrometry, machine learning, MHC, sequence motif

\section{Abbreviations: \\ FDR False discovery rate \\ HLA Human leukocyte antigen \\ PSM Peptide-spectrum match}

Word count: 4845 


\begin{abstract}
Liquid chromatography tandem mass spectrometry (LC-MS/MS) has become the standard platform for the characterization of immunopeptidomes, the collection of peptides naturally presented by MHC molecules to the cell surface. The protocols and algorithms used for immunopeptidomics data analysis are based on tools developed for traditional bottom-up proteomics, that address the identification of peptides generated by tryptic digestion. Such algorithms are generally not tailored to the specific requirements of MHC ligand identification and, as a consequence, immunopeptidomics datasets suffer from dismissal of informative spectral information and high false discovery rates. Here, we propose a new pipeline for the refinement of peptide-spectrum matches (PSM), based on the assumption that immunopeptidomes contain a limited number of recurring peptide motifs, corresponding to MHC specificities. Sequence motifs are learned directly from the individual peptidome by training a prediction model on high-confidence PSMs. The model is then applied to PSM candidates with lower confidence, and sequences that score significantly higher than random peptides are rescued as likely true ligands. We applied the pipeline to MHC class I immunopeptidomes from three different species, and showed that it can increase the number of identified ligands by up to $20-30 \%$, while effectively removing false positives and products of co-precipitation. Spectral validation using synthetic peptides confirmed the identity of a large proportion of rescued ligands in the experimental peptidome.
\end{abstract}




\section{Statement of significance}

Characterizing immunopeptidomes with high fidelity and depth is essential to further our understanding of the rules of antigen presentation in healthy, infected or cancerous cells. While mass spectrometry has become an invaluable platform to accurately sequence immunopeptidomes, the protocols and algorithms for peptide identification do not yet take advantage of the characteristic length distribution and particular sequence features of MHC ligands. Here, we propose a new pipeline for the refinement of spectrum-peptide assignment, designed specifically for $\mathrm{MHC}$ ligand identification. By modeling the peptidome as a collection of a limited number of specificities, corresponding to the MHC alleles of the cell line, our method achieves increased sequencing depth, while at the same time removing potential experimental outliers and contaminants. 


\section{Introduction}

Major Histocompatibility Complex (MHC) molecules are an essential component of the immune system. The set of peptides presented by MHC molecules on the cell surface, collectively known as the immunopeptidome, is a unique fingerprint of the state of a cell and enables immune surveillance by T-cells against pathogens and cancer. Liquid chromatography tandem mass spectrometry (LC-MS/MS) has become the method of choice to characterize and quantify immunopeptidomes in recent years ${ }^{[1-4]}$.

In most LC-MS/MS pipelines for the identification of MHC ligands, spectra from eluted peptides are matched against a reference protein database using algorithms like SEQUEST ${ }^{[5]}$, MaxQuant ${ }^{[6]}$ or PEAKS ${ }^{[7]}$, and subsequently filtered against a decoy database to limit the false discovery rate (FDR) using a probability scoring approach. Strict FDR filters (typically in the order of 1\%) should ensure that most spectra are correctly assigned to bona fide ligands, but often leads to discarding a large portion of spectra with high content of information. At the same time, while $99 \%$ of peptidespectrum matches (PSM) are theoretically correct when fixing a FDR=1\%, a proportion of sequenced peptides may originate from co-precipitating proteins, such as ribosomal or histone proteins ${ }^{[8]}$. Furthermore, some peptide identifications can result from carryover on the chromatographic columns being used ${ }^{[9]}$. Such observations can usually be corrected in traditional proteomics analyses, where multiple peptides contribute to the final protein identification and quantitation. On the contrary, in immunopeptidomics data every single peptide identification is relevant to contribute to the final dataset ${ }^{[10]}$.

A few approaches have been proposed to increase the depth of sequenced immunopeptidomes, i.e. the number of confident peptide-spectrum assignments of a given experiment. For example, Percolator ${ }^{[11]}$ performs machine learning on highconfidence matches to rescore database search results for lower-confidence peptides. For each peptide-spectrum match (PSM), Percolator computes a vector of features related to the quality of the match, for example mass deviations or PSM quality scores ($10 \log \mathrm{P}$ scores in the case of PEAKS ${ }^{[7]}$, or PEP scores in MaxQuant ${ }^{[12]}$, to train a support vector machine classifier. These features are then used to re-score other peptides that previously fell below the minimum confidence level. Note that Percolator does not at any point exploit the actual primary sequence of the peptides, but rather more abstract features of each PSM. The SpectMHC program ${ }^{[13]}$ uses a different 
strategy: instead of matching spectra to an entire protein database, it does construct reduced, targeted databases of potential MHC ligands. By limiting the size of the reference proteome, SpectMHC also reduces the amount of spurious decoy hits. While reasonable for common $\mathrm{MHC}$ alleles with well-characterized binding preferences, this approach would fail for alleles having poorly characterized motifs. Moreover, part of the appeal of using LC-MS/MS for the identification of MHC ligands is that it is an unsupervised and unbiased approach; in contrast to a bias being introduced when prior knowledge of the expected binding motifs is imposed to the reference proteome ${ }^{[14]}$.

In this work, we propose a new approach, MS-rescue, for rescoring PSMs below a $1 \%$ FDR cut-off, specifically tailored to MHC ligand sequencing. Our pipeline uses highconfidence peptide identifications obtained from a full-database search (i.e. peptides with PSM scores above a given FDR threshold) to construct a model of the MHC binding specificities contained in the sample, accounting for the binding and length preferences of the specific peptidome. The model is then applied to rescore lower confidence peptides - with scores below the FDR threshold - and identify candidate ligands that are congruent with the motifs learned from the high-confidence peptides. Using MHC class I peptidomic data from three different species, we investigate the impact of our rescoring approach on the size and quality of sequenced immunopeptidomes.

\section{Materials and Methods}

\subsection{Cell lines}

TC-1 and Jurkat cells were cultured in RPMI 1640 (Sigma) supplemented with 10\% heat-inactivated fetal calf serum, $2 \mathrm{mM}$ L-glutamine, $1 \mathrm{mM}$ pyruvate, $0.1 \mathrm{mM}$ NonEssential Amino Acids Solution and $100 \mathrm{U}$ penicillin/ml, and was incubated at $37^{\circ} \mathrm{C}$ in $5 \%$ CO2 (9). TC-1 cells were harvested by trypsinization, washed twice, and resuspended at $0.5 \times 10^{6} / \mathrm{ml}$ in PBS. Groups of 6 mice (C57BL/6 six-week-old females purchased from Harlan, UK) were challenged subcutaneously with $5 \times 10^{4}$ TC-1 cells per mouse resuspended in $100 \mathrm{~mL}$ of PBS and injected into the right rear flank. Tumour diameters were measured in two orthogonal dimensions using electronic digital callipers. Tumour

volumes were calculated from these measurements according to: (width ${ }^{2} \mathrm{x}$ length) $\mathrm{x}$ 0.52 . The mice were euthanized once the tumours exceeded a diameter of $10 \mathrm{~mm}$ in any one direction or became necrotic. 
Mouse care and experimental procedures were carried out in accordance with the terms of the UK Animals (Scientific Procedures) Act Project License (PPL 30/2947) and approved by the University of Oxford Animal Care and Ethical Review Committee.

\subsection{HLA-associated peptide purification}

TC-1 tumours were homogenized in $1 \mathrm{ml} 2 \mathrm{x}$ lysis buffer (1\% Igepal, $300 \mathrm{mM}$ sodium chloride, $100 \mathrm{mM}$ Tris, $\mathrm{pH}$ 8.0) supplemented with protease inhibitor cocktail (Roche) using a bead beater (Precellys 24 bead-beater, Bertin Technologies) five times for $10 \mathrm{~s}$ at $6500 \mathrm{rpm}$. Jurkat cells were resuspended in $1 \mathrm{ml} 2 x$ lysis buffer per $10^{8}$ cells. Lysates were cleared by subsequent centrifugation steps at $500 \mathrm{~g}$ for $10 \mathrm{~min}$ and then $20,000 \mathrm{~g}$ for $60 \mathrm{~min} .1 \mathrm{mg}$ per sample of anti-mouse H-2-K $/ \mathrm{H}-2-\mathrm{D}^{\mathrm{b}}$ antibody (ATCC HB-51) or anti-HLA class I antibody (W6/32, ATCC HB-95), was bound and cross-linked to $1 \mathrm{ml}$ Protein A beads (GE healthcare) and used for immunoprecipitation of HLA complexes. In brief, lysates were incubated with the antibody beads over night at $4^{\circ} \mathrm{C}$ in $1 \mathrm{x}$ lysis buffer and washed subsequently with 10 bed volumes of $50 \mathrm{mM}$ Tris, $\mathrm{pH} 8.0$ containing either $150 \mathrm{mM}, 450 \mathrm{mM}$ and finally no salt. Peptides were eluted with $5 \mathrm{ml}$ of $10 \%$ acetic acid. Dried peptides were suspended and injected onto a 4.6 × $50 \mathrm{~mm}$ ProSwift RP-1S column (Thermo Fisher Scientific). Peptides were separated from larger complex components by elution using a $500 \mu \mathrm{l} / \mathrm{min}$ flow rate over $10 \mathrm{~min}$ from $2-34 \% \mathrm{ACN}$ in $0.1 \%$ TFA. Alternate fractions that did not contain the beta-2-microglobulin were pooled and two final fractions were analyzed by nUPLC-MS2.

\subsection{Nano-ultra performance liquid chromatography tandem mass spectrometry (nUPLC-MS2)}

HLA peptides eluted from tissues were separated on an Ultimate 3000 RSLCnano system (Thermo Scientific) using a PepMap C18 column, $2 \mu \mathrm{m}$ particle size, $75 \mu \mathrm{m} \times 50$ $\mathrm{cm}$ (Thermo Scientific) with a $1 \mathrm{~h}$ linear gradient of $3-25 \%$ buffer B $(0.1 \%$ formic acid, $5 \%$ DMSO in acetonitrile) in buffer A (0.1\% formic acid, $5 \%$ DMSO in water) at a flowrate of $250 \mathrm{nl} / \mathrm{min}$. Peptides were introduced using an Easy-Spray source at $2000 \mathrm{~V}$ and to a Fusion Lumos (Thermo Scientific). The ion transfer tube temperature was set to $305^{\circ} \mathrm{C}$. Full MS spectra were recorded from $300-1500 \mathrm{~m} / \mathrm{z}$ in the Orbitrap at 120,000 resolution with an AGC target of 400,000. Precursor selection was performed using TopSpeed mode at a cycle time of $2 \mathrm{~s}$. Peptide ions were isolated using an isolation width of 1.2 
amu and trapped at a maximal injection time of 120 ms with an AGC target of 300,000 . Higher-energy collisional dissociation (HCD) fragmentation was induced at an energy setting of 28 for peptides with a charge state of $2-4$, while singly charged peptides were fragmented at an energy setting of 32 at lower priority. Fragments were analysed in the Orbitrap at 30,000 resolution.

\subsection{Data analysis}

Analysis of raw data was performed using Peaks 8.5 software (Bioinformatics Solutions). Sequence interpretation of $\mathrm{MS}^{2}$ spectra was carried out using databases as detailed below. Peptides with a length of less than 7 amino acids were excluded from the analysis results and peptides with a Peaks score of below 5 were ignored. The false discovery rate was estimated with randomized decoy database searches within Peaks. Details of databases: Human dataset: SwissProt Homo sapiens entries, taxon identifier 9606, downloaded on 18/5/2017; 42,221 entries. Mouse dataset: SwissProt mus musculus entries, taxon identifier 10090, downloaded on 17/5/2018; 16,978 entries. The mass spectrometry data sets have been deposited to the ProteomeXchange Consortium ${ }^{[15]}$ via the PRIDE partner repository with the identifier PXD008151 (bovine dataset), PXD011723 (human dataset), and PXD011794 (mouse dataset).

\section{Results and Discussion}

\subsection{The MS-rescue pipeline}

The essential input to the program consists of a list of peptide assignments with associated confidence scores, and a score threshold separating high- and lowconfidence hits. Search engines for peptide identification normally provide the threshold on the confidence values (-10logP in the case of PEAKS, PEP scores in the case of MaxQuant) that corresponds to a desired FDR, e.g. FDR=1\%. High-confidence hits constitute the "training set" for the method, whereas low-confidence hits are placed in a "recovery set" (Figure 1a). The training set is then submitted to GibbsCluster ${ }^{[16]}$, which groups the peptides into a finite number of clusters. GibbsCluster attempts to group similar peptides together, while discarding to the "trash cluster" those sequences that cannot be clustered with other peptides. The basic assumption is that peptide sequences in a single immunopeptidomics experiment should cluster into a finite number 
of sequence motifs (up to six for HLA class I), corresponding to the binding motifs of the MHC molecules expressed by the cell line. Sequences that cannot be matched to any conserved specificity are likely to be false positives or contaminants and are therefore assigned to the "trash cluster" - and discarded from further analysis (Figure 1b). We have previously shown that this approach is effective for the detection of outliers in immunopeptidomics data sets ${ }^{[9]}$. Peptides that pass the trash filter are then complemented with a negative set of $10^{*} \mathrm{~N}$ random peptides for each length $8-13$, where $\mathrm{N}$ is the number of peptides for the most represented peptide length. This set of positive and negative examples is used to train a NNAlign model ${ }^{[17]}$ (Figure 1c). Given a training set of peptide sequences with associated target values, NNAlign generates a model of the sequence motif(s) that are overrepresented in the positive training examples ${ }^{[17,18]}$. In the context of the MS-rescue pipeline, NNAlign constructs a model that should capture the MHC motifs contained in the high-confidence hits of the specific sample. The initial grouping by GibbsCluster is preserved by tagging each sequence according to its cluster with a single letter in the set $\{A, G, S, V, T, P\}$ - for example, in the case of two clusters, all peptides in cluster 1 are assigned the tag $A$ and peptides in cluster 2 are assigned the tag G. The tags are presented to NNAlign as part of the input, similarly to the pseudosequences used in NetMHCpan ${ }^{[19]}$, and allow the neural networks to incorporate clusterspecific features, such as peptide length distributions. The NNAlign model can then be used to evaluate all peptides in the "recovery set", and obtain a prediction score for each peptide (Figure 1d). The model scores are reported as percentile rank scores (\%Rank), calculated by comparing the prediction scores to the background distribution of scores expected for the model for the given group tag, estimated by running 100,000 random peptides equally distributed over the lengths 8 to 13. Peptides in the recovery set that score among the top \%Rank scores (e.g. 1\%) are "rescued" from the low-confidence hits and added to the list of identified ligands (Figure 1e).

The pipeline is distributed as a package (MS-rescue-1.0) and is completely automated it only requires a list of peptides with associated scores, and the threshold that identifies high-confidence hits. Several other parameters can optionally be specified, including the minimum and maximum peptide length to be included in the analysis, the parameters for GibbsCluster (threshold for the trash cluster, number of iterations and initial seeds), the number of random negatives per positive example, and the architecture of the NNAlign models (number of training iterations, hidden neurons, initial seeds). The program returns an evaluation of the low-confidence PSMs in the sample, based on the sequence 
motifs identified in the training set of high-confidence peptides. Each evaluation data point receives a percentile score, which describes how well the peptide fits in the motifs learned from the high-confidence hits. For example, a percentile score of $1 \%$ means that the peptide matches one of the motifs as well or better than $99 \%$ of any random natural sequence of length 8-13. Therefore, the lower the \%Rank score the more likely that a given peptide is a genuine ligand for one of the MHC molecules in the sample. In the following section, we illustrate the application of the MS-rescue pipeline for the rescoring of LC-MS/MS ligand datasets from three different species.

\subsection{Rescoring peptidomes from human, mouse and cattle}

We aimed to validate the MS-rescue pipeline on three MHC class I immunopeptidomics data sets from different species (Table 1). The A18-Tpm (bovine) data set has been previously published [20]; the Jurkat (human) and TC-1 (murine) peptidomes were sequenced for this study (see Materials and Methods). Because the large majority of MHC class I ligands have length between 8 and 13 amino acids ${ }^{[21]}$, we limited the analyses to peptides in this range of length. The number of $8-13 \mathrm{mer}$ ligands that passed the FDR 1\% filter was 8007 for the Jurkat cell line, 3524 for TC-1, and 6829 for the A18_Tpm data set; the number of peptides to be evaluated in the recovery set was 9655,6621 and 3695 for the three cell lines, respectively (Table 1). The GibbsCluster analysis detected the correct number of specificities for the TC-1 set and A18-Tpm, while it underestimated the number of alleles in the Jurkat data set (Supplementary Figure 1). As has been previously reported, unsupervised clustering tends to underestimate the number of specificities when multiple MHCs have redundant motifs and/or low expression levels ${ }^{[16,22]}$. In the case of the Jurkat cell line, the expressed HLA$B$ alleles $B^{*}$ 07:02 and $B^{*} 35: 03$ belong to the $B 07$ supertype and are known to have nearly identical peptide-binding preferences ${ }^{[23,24]}$; unsupervised clustering therefore fails to separate them into two separate specificities. Finally, the fraction of peptides captured by the "trash cluster" varied between $2.6 \%$ (A18-Tpm) to $7.9 \%$ (TC-1), suggesting a level of variability in the quality of the different data sets.

The NNAlign models trained on the high-confidence hits (filtered and tagged by GibbsCluster, and enriched with a flat distribution of random negatives as described above) consistently recovered a substantial fraction of high-ranking peptides. Setting a percentile Rank score threshold of $1 \%$, the method recovers 2578, 938 and 960 for the 
three data sets, respectively. This corresponds to an increase of $32 \%, 27 \%$ and $14 \%$, respectively, in the size of the sequenced peptidome, compared to the amount of peptides selected solely on the FDR $1 \%$ filter. Using a more conservative threshold of Rank $=0.2 \%$, the number of rescued peptides in the three sets is 1368,587 and 552 , corresponding to an increase of $17 \%, 17 \%$ and $8 \%$, respectively.

\subsection{Validation of peptides recovered below the FDR threshold}

The NNAlign models generated by the MS-rescue pipeline are conceptually very similar to NetMHCpan, a popular method for the prediction of peptide-MHC interactions ${ }^{[25]}$. In fact, NNAlign is the machine-learning engine at the core of many pieces of software in the NetMHC family ${ }^{[17,26]}$. The essential difference is that NetMHCpan is trained on data from multiple sources, whereas the NNAlign models generated by the MS-rescue pipeline are trained directly and exclusively on the ligands from the specific peptidome that is subject of the analysis. As an alternative approach to the construction of samplespecific models using GibbsCluster and NNAlign (Figure 1b-1c), we considered rescoring low-confidence peptides directly using NetMHCpan. To assess similarities and differences in the two approaches, we calculated NetMHCpan-4.0 \%Rank scores for the Jurkat recovery set, and compared the results with the NNAlign \%Rank scores obtained with the automated pipeline. We observed a strong correlation $(P C C=0.76)$ between the Rank scores of the two methods (Figure 2a), with a large overlap between the peptides with ranks in the top $0.2 \%$ (Figure $2 \mathrm{~b}$ ), as well as top $1 \%$ (Figure $2 \mathrm{c}$ ).

In order to validate our findings, we designed seven sectors corresponding to different \%Rank intervals of NNAlign and NetMHCpan (Figure 2a), and selected a pool of peptides from the Jurkat recovery set that fell in these sectors. A total of 100 peptides (40 from sector A, 10 for each of the remaining sectors) were synthesized and analysed under identical MS acquisition conditions. Resulting spectra were then compared to the spectra acquired in the experiment with the regarding sequence interpretation to confirm or reject the peptide identity (Figure 3 ). We found that over $70 \%$ of the peptides in sectors $A, B$ and $D$ could be confirmed by spectral validation, as well as $50 \%$ from sector C (Figure 2d). Conversely, only few peptides (4 out of 30 ) from sectors $E, F$ and $G$ could be confirmed. Note that the peptides in these groups are instances for which NNAlign and NetMHCpan dramatically disagree, being ranked high by one method and very low by the other. However, they represent a small minority of the predictions: for example, of 
all peptides with predicted NNAlign rank $\leq 0.2 \%, 97 \%$ of the peptides are found in sectors $A$ or $B$, whereas only about $3 \%$ are in sector $E$. Therefore, because the MSrescue pipeline recovers PSMs based solely on NNAlign \%Rank, its overall precision $P$ $=\mathrm{TP} /(\mathrm{TP}+\mathrm{FP})$ including all tested peptides with NNAlign \%Rank below $0.2 \%$ would remain around $80 \%$, as found in sectors $A$ and $B$.

\section{Concluding Remarks}

In summary, MS-rescue is a fully automated pipeline for the rescoring of LC-MS/MS peptide-spectrum assignments from immunopeptidomics data sets. The approach is self-contained, in that it constructs a model directly on the peptidome under analysis and uses no external information about the expected binding motifs. The workflow is applied post hoc to any list of peptide assignments generated by PEAKS or other search engines for MS data analysis, and returns a rescored list of candidate ligands. Two main classes of peptides are affected by the rescoring: 1) outliers, contaminants and potential products of co-precipitation found among the high-confidence hits; and 2) peptides below the FDR threshold, but that match closely the motifs learned from high-confidence hits. In our framework, peptides in the first category are removed from the list of identifications, while peptides in the second category are "rescued" as bona fide ligands. In this work, we showed how this approach effectively improves the number of confident peptide identifications in LC-MS/MS ligand elution experiments. Increasing the quality and depth of immunopeptidomes has important implications for more accurate definition of peptide-MHC binding motifs, as well as for the identification of cancer neo-epitopes and pathogen-derived $\mathrm{T}$ cell epitopes. The MS-rescue pipeline is distributed as a package and available for download at http://www.cbs.dtu.dk/cgibin/sw request?msrescue 


\section{Acknowledgements}

The TC-1 cell line was kindly provided to Professor Lucy Dorrell by Professor T. C. Wu,

Johns Hopkins University, Baltimore, Maryland, USA. MN is a researcher at the Argentinean Research Council (CONICET); MA is funded by a postdoctoral research grant from CONICET.

The authors have declared no conflict of interest. 


\section{References}

[1] D. F. Hunt, R. A. Henderson, J. Shabanowitz, K. Sakaguchi, H. Michel, N. Sevilir, A. L. Cox, E. Appella, V. H. Engelhard, Science 1992, 255, 1261.

[2] K. Falk, O. Rötzschke, S. Stevanović, G. Jung, H.-G. Rammensee, J. Immunol. Baltim. Md 1950 2006, 177, 2741.

[3] E. Caron, D. J. Kowalewski, C. Chiek Koh, T. Sturm, H. Schuster, R. Aebersold, Mol. Cell. Proteomics MCP 2015, 14, 3105.

[4] M. Bassani-Sternberg, E. Bräunlein, R. Klar, T. Engleitner, P. Sinitcyn, S. Audehm, M. Straub, J. Weber, J. Slotta-Huspenina, K. Specht, M. E. Martignoni, A. Werner, R. Hein, D. H Busch, C. Peschel, R. Rad, J. Cox, M. Mann, A. M. Krackhardt, Nat. Commun. 2016, 7, 13404.

[5] J. K. Eng, A. L. McCormack, J. R. Yates, J. Am. Soc. Mass Spectrom. 1994, 5, 976.

[6] J. Cox, M. Mann, Nat. Biotechnol. 2008, 26, 1367.

[7] J. Zhang, L. Xin, B. Shan, W. Chen, M. Xie, D. Yuen, W. Zhang, Z. Zhang, G. a. Lajoie, B. Ma, Mol. Cell. Proteomics 2012, 11, M111.010587.

[8] D. Mellacheruvu, Z. Wright, A. L. Couzens, J.-P. Lambert, N. A. St-Denis, T. Li, Y. V. Miteva, S. Hauri, M. E. Sardiu, T. Y. Low, V. A. Halim, R. D. Bagshaw, N. C. Hubner, A. Al-Hakim, A. Bouchard, D. Faubert, D. Fermin, W. H. Dunham, M. Goudreault, Z.-Y. Lin, B. G. Badillo, T. Pawson, D. Durocher, B. Coulombe, R. Aebersold, G. Superti-Furga, J. Colinge, A. J. R. Heck, H. Choi, M. Gstaiger, S. Mohammed, I. M. Cristea, K. L. Bennett, M. P. Washburn, B. Raught, R. M. Ewing, A.-C. Gingras, A. I. Nesvizhskii, Nat. Methods 2013, 10, 730.

[9] B. Alvarez, C. Barra, M. Nielsen, M. Andreatta, Proteomics 2018, 18, e1700252.

[10]P. Faridi, A. W. Purcell, N. P. Croft, Proteomics 2018, 18, e1700464.

[11]L. Käll, J. D. Canterbury, J. Weston, W. S. Noble, M. J. MacCoss, Nat. Methods 2007, 4, 923.

[12]S. Tyanova, T. Temu, J. Cox, Nat. Protoc. 2016, 11, 2301.

[13]J. P. Murphy, P. Konda, D. J. Kowalewski, H. Schuster, D. Clements, Y. Kim, A. M. Cohen, T. Sharif, M. Nielsen, S. Stevanovic, P. W. Lee, S. Gujar, J. Proteome Res. 2017, 16, 1806.

[14]A. Sticker, L. Martens, L. Clement, Nat. Methods 2017, 14, 643.

[15]J. A. Vizcaíno, E. W. Deutsch, R. Wang, A. Csordas, F. Reisinger, D. Ríos, J. A. Dianes, Z. Sun, T. Farrah, N. Bandeira, P.-A. Binz, I. Xenarios, M. Eisenacher, G. Mayer, L. Gatto, A. Campos, R. J. Chalkley, H.-J. Kraus, J. P. Albar, S. MartinezBartolomé, R. Apweiler, G. S. Omenn, L. Martens, A. R. Jones, H. Hermjakob, Nat. Biotechnol. 2014, 32, 223.

[16]M. Andreatta, B. Alvarez, M. Nielsen, Nucleic Acids Res. 2017, DOI 10.1093/nar/gkx248.

[17]M. Nielsen, M. Andreatta, Nucleic Acids Res. 2017, DOI 10.1093/nar/gkx276.

[18]M. Andreatta, C. Schafer-Nielsen, O. Lund, S. S. S. Buus, M. Nielsen, PLoS ONE 2011, 6, e26781.

[19]M. Nielsen, C. Lundegaard, T. Blicher, K. Lamberth, M. Harndahl, S. Justesen, G. Røder, B. Peters, A. Sette, O. Lund, S. Buus, PloS One 2007, 2, e796.

[20]M. Nielsen, T. Connelley, N. Ternette, J. Proteome Res. 2018, 17, 559.

[21]T. Trolle, C. P. McMurtrey, J. Sidney, W. Bardet, S. C. Osborn, T. Kaever, A. Sette, W. H. Hildebrand, M. Nielsen, B. Peters, J. Immunol. Baltim. Md 1950 2016, 196, 1480.

[22]M. Bassani-Sternberg, D. Gfeller, J. Immunol. Baltim. Md 1950 2016, 197, 2492.

[23]J. Sidney, B. Peters, N. Frahm, C. Brander, A. Sette, BMC Immunol. 2008, 9, 1. 
[24]M. Thomsen, C. Lundegaard, S. Buus, O. Lund, M. Nielsen, Immunogenetics 2013, $65,655$.

[25]V. Jurtz, S. Paul, M. Andreatta, P. Marcatili, B. Peters, M. Nielsen, J. Immunol. Baltim. Md 1950 2017, 199, 3360.

[26]M. Andreatta, M. Nielsen, Methods Mol. Biol. Clifton NJ 2018, 1785, 269. 


\section{Figure Legends}

Figure 1: The MS-rescue workflow for rescoring MHC ligands detected by massspectrometry. a) A specified FDR threshold (typically 1\%) is used to separate MS/MS spectra of MHC ligands into high-confidence hits and low-confidence hits. b) High-confidence hits are submitted to GibbsCluster, which groups the peptides into $\mathrm{N}$ clusters. Outliers and potential contaminants are automatically collected into a "trash cluster" and removed from analysis. c) Peptides passing the GibbsCluster filter are tagged by their cluster number and pooled to train a NNAlign neural network model. d) The NNAlign model is then applied on the evaluation set to obtain prediction scores for each low-confidence peptide. e) The prediction scores are compared to the background score distribution of the NNAlign model (calculated on a large set of random natural sequences) to derive percentile Rank scores. Peptides with \%Rank scores below a given threshold are "rescued" from the low confidence hits and added to the list of identified ligands.

Figure 2: Model predictions on the Jurkat recovery set. a) Number of peptides from the recovery set predicted within discrete \%Rank intervals for NNAlign and NetMHCpan-4.0. Peptides were selected for validation from seven sectors (A to $G$ ), corresponding to different $\%$ Rank intervals. b) Number of peptides in the recovery set with predicted rank score $\leq 0.2 \%$ for NNAlign and NetMHCpan. The shaded area corresponds to sector A. c) Number of peptides in the recovery set with predicted rank score $\leq 1 \%$ for the two methods. The shaded area corresponds to sectors $A$ to $D$. d) Percentage of peptides derived from sectors $A$ to $G$ that were confirmed by spectral validation.

Figure 3: Spectral validation. Fragment spectra for indicated peptide sequences. The detected mass over charge ratio of the charged peptide precursor is stated above (for experimental) or below (for synthetic) each spectrum. All fragment ions that were detected and assigned are indicated in the peptide sequence, most abundant fragment ions are assigned in the spectrum. Fragment ions are annotated as follows: $b$ : $\mathrm{N}$-terminal fragment ion; $\mathrm{b}^{++}$: doubly charged $\mathrm{N}$ terminal peptide ion; $\mathrm{y}$ : C-terminal fragment ion; $\mathrm{y}^{++}$: doubly charged $\mathrm{C}$-terminal peptide ion; pre $^{++}$: doubly charged precursor ion.

Supplementary Figure 1: GibbsCluster analysis of the Jurkat (a), A18_Tpm (b) and TC-1 (c) peptidomes. The motif logos correspond to the optimal clustering solution identified by GibbsCluster. For reference, compare to the Motif Viewer of NetMHCpan for individual MHC molecules at: http://www.cbs.dtu.dk/services/NetMHCpan/logos_ps.php 


\section{Tables}

Table 1: Properties and result summary of the data sets used for validation.

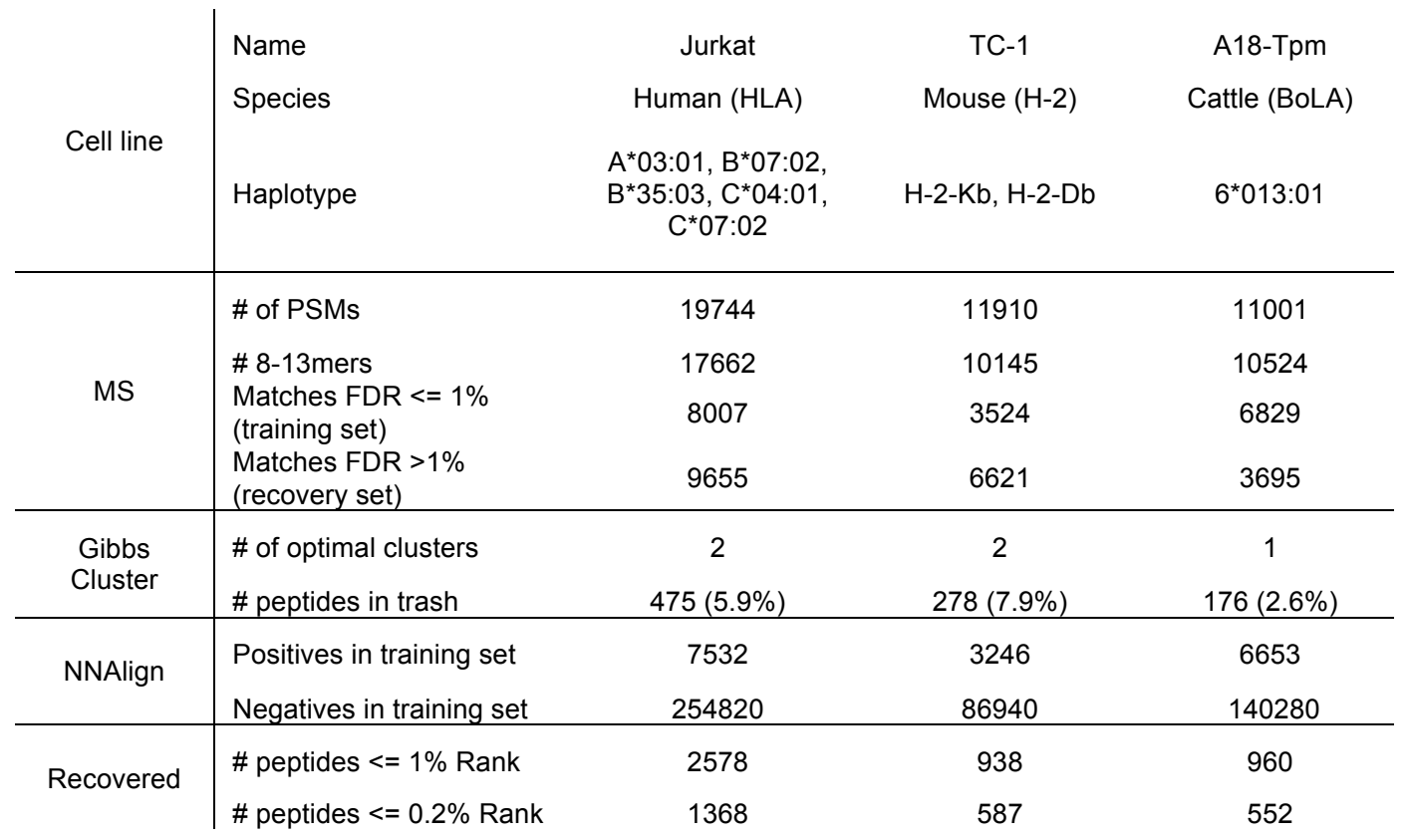


Figure 1

a)

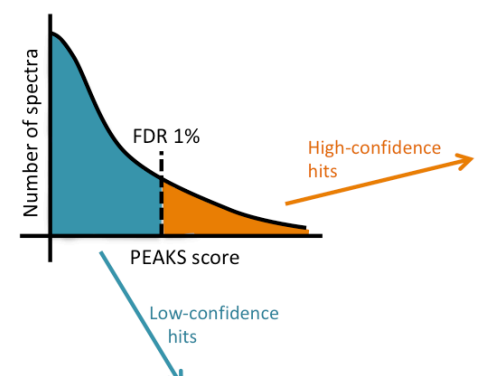

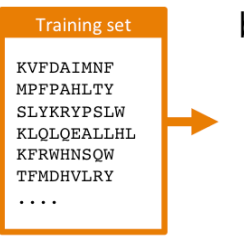

c)

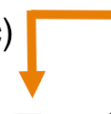

b)
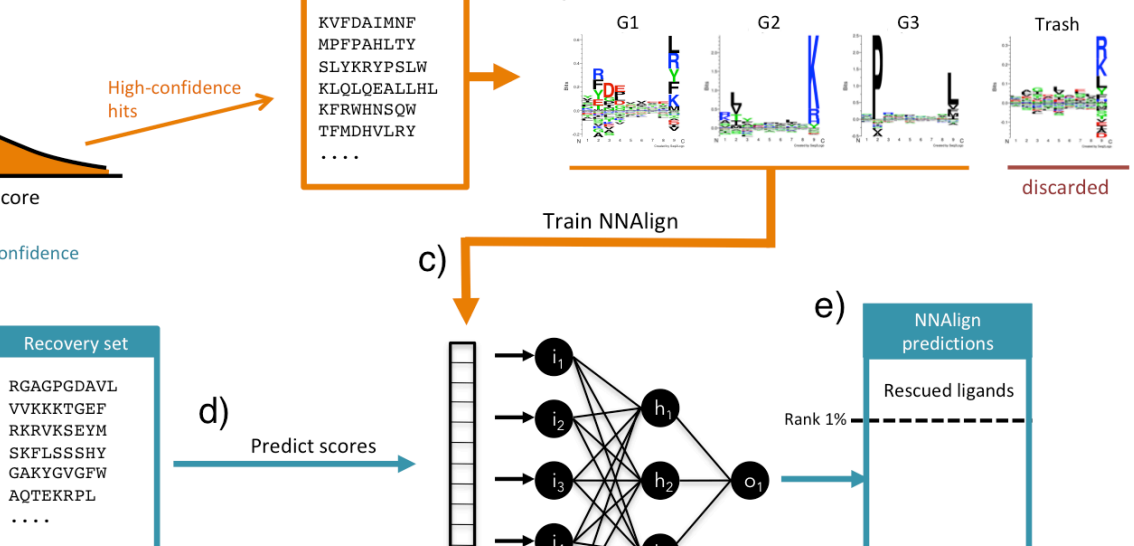

d)

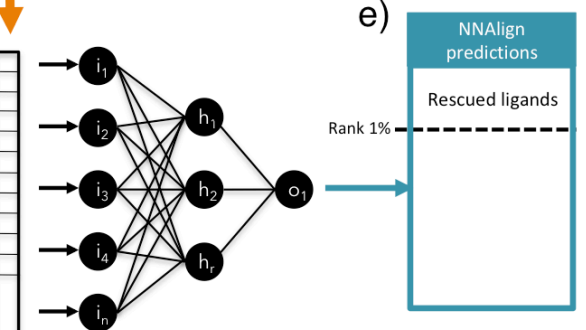


Figure 2

a)

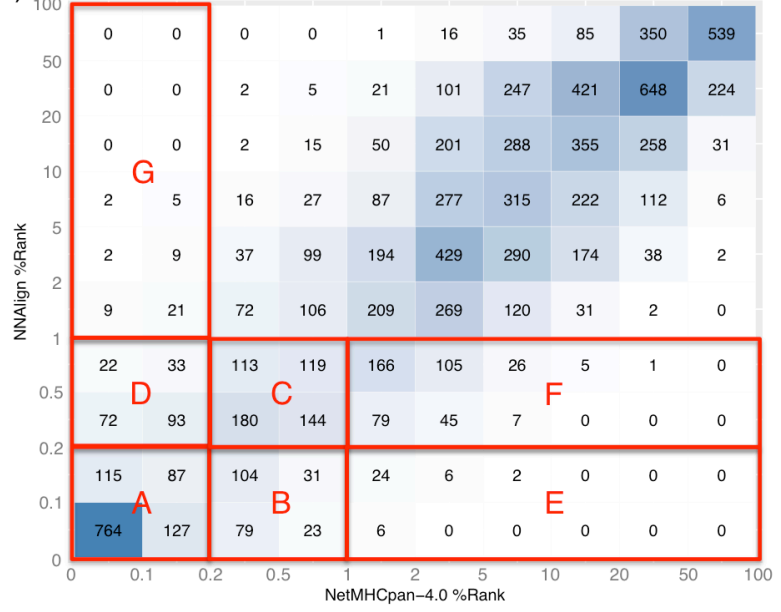

b)

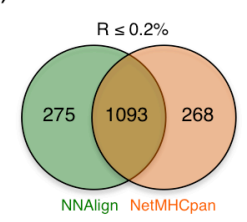

c)

d)

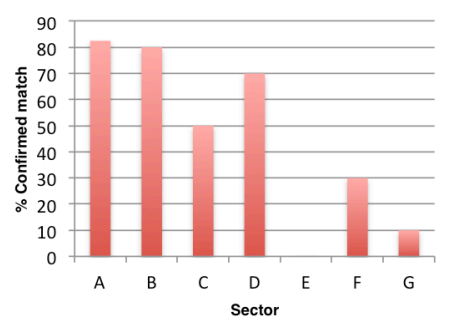


Figure 3
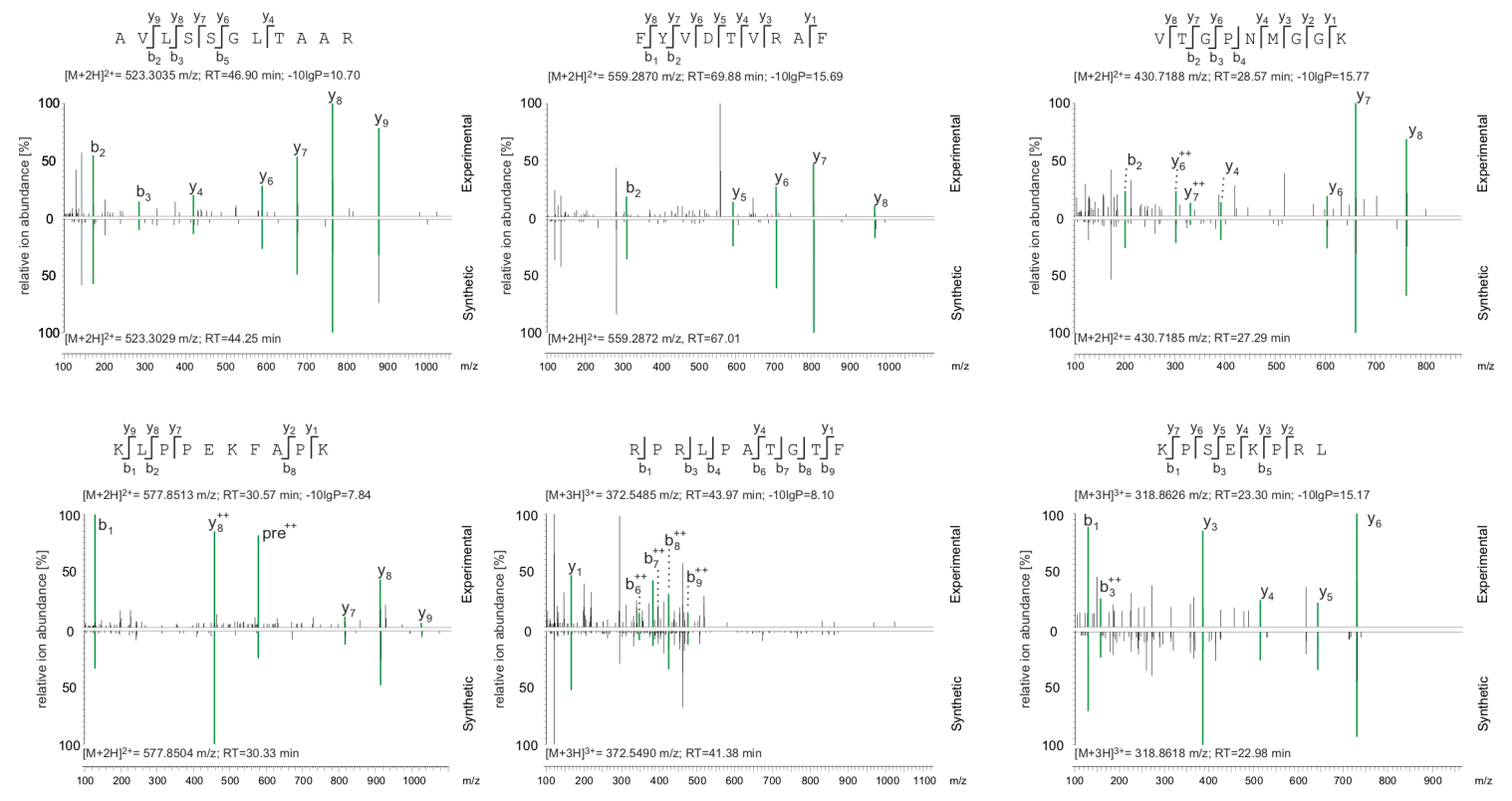


\section{Supplementary Figure S1}

a)

Cells: Jurkat (HLA)

Haplotype: $A^{*} 03: 01$,

$B^{*} 07: 02, B^{\star} 35: 03$,

$C * 04: 01, C * 07: 02$

Group1: 2977 pep

Group2: 4555 pep

Trash: 475 pep
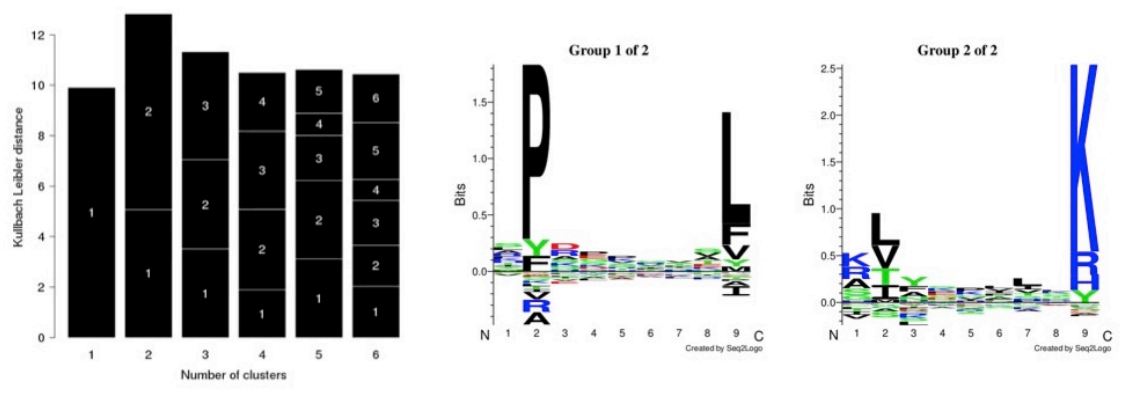

b)

Cells: A18 Tpm (BoLA)

Haplotype: $6^{\star}$ 013:01

Group1: 6653 pep

Trash: 176 pep
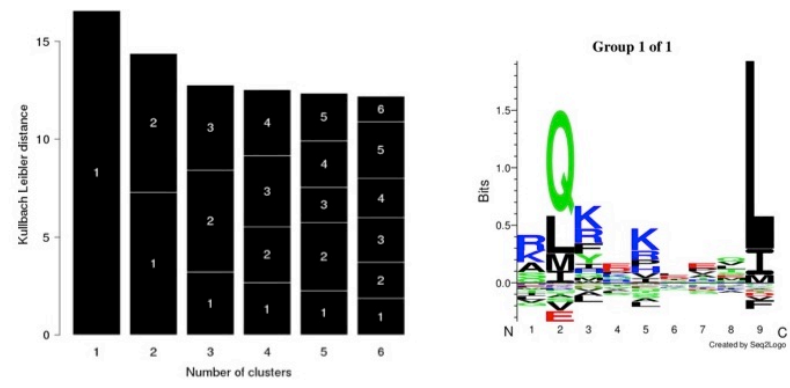

c)

Cells: TC-1 $(\mathrm{H}-2)$

Haplotype: $\mathrm{H}-2-\mathrm{Kb}$,

$\mathrm{H}-2-\mathrm{Db}$

Group1: 1602 pep

Group2: 1644 pep

Trash: 278 pep
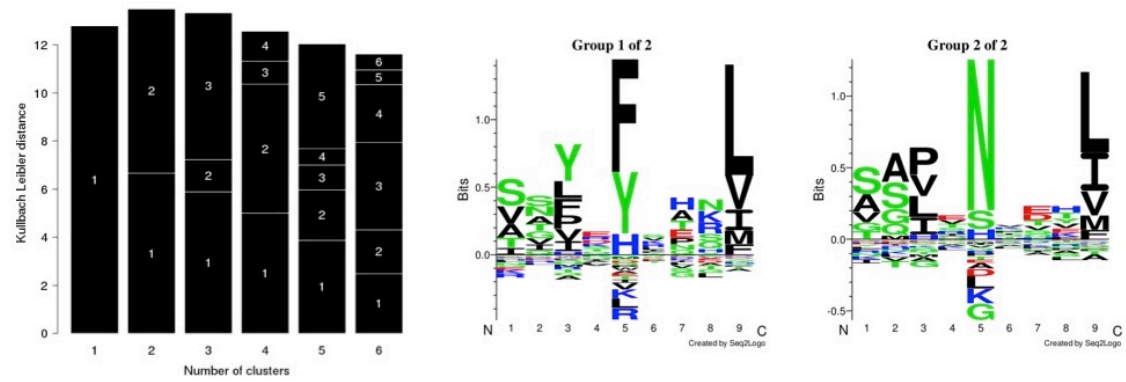